\title{
Global and local tension measurements in biomimetic skeletal muscle tissues reveals early mechanical homeostasis
}

Arne D. Hofemeier ${ }^{1}$, Tamara Limon ${ }^{1}$, Till M. Muenker ${ }^{1}$, Bernhard Wallmeyer ${ }^{1}$, Alejandro Jurado ${ }^{1}$, Mohammad E. Afshar ${ }^{2,3}$, Majid Ebrahimi ${ }^{2,3}$, Penney M. Gilbert ${ }^{2-4}$, Timo Betz ${ }^{1, *}$

1 Institute for Cell Biology, University of Münster, Münster, Germany 2 Institute of Biomaterials and Biomedical Engineering, University of Toronto, Toronto, Canada

3 Donnelly Centre, University of Toronto, Toronto, Canada 4 Department of Cell and Systems Biology, University of Toronto, Toronto, Canada

\section{* timo.betz@uni-muenster.de}

\section{Abstract}

The mechanical properties and tension of muscle tissue are tightly related to proper skeletal muscle function, which makes experimental access to the biomechanics of muscle tissue development a key requirement to advance our understanding of muscle function and development. Recently developed elastic in vitro culture chambers allow for raising 3D muscle tissue under controlled conditions and measurements of tissue force generation. However, these chambers are inherently incompatible with high resolution microscopy limiting their usability to global force measurements, and preventing the exploitation of modern fluorescence based investigation methods for live and dynamic measurements. Here we present a new chamber design pairing global force measurements, quantified from post deflection, with local tension measurements obtained from elastic hydrogel beads embedded in the muscle tissue. High resolution 3D video microscopy of engineered muscle development, enabled by the new chamber, shows an early mechanical tissue homeostasis that remains stable in spite of continued myotube maturation.

\section{Introduction}

Skeletal muscle is one of the most abundant tissues in the human body and is crucial for essential functions such as limb movement, thermogenesis and maintaining posture $9,26,38$. Skeletal muscle atrophy is debilitating and is a typical outcome of diseases like muscular dystrophies but also aging. Characterizing the biomechanics of muscle tissue is a key element to understand both, muscle development and muscle degeneration. While this characterization has been largely achieved on the global tissue level, the local mechanical properties during muscle development and maturation remain inaccessible. This is largely due to the limited optical access of muscle tissue which prevents the use of modern fluorescence microscopy based methods. Indeed, molecular biology has contributed a phlethora of fluorescence microscopy based tools to label molecules [6,56, modify signaling cascades [16] and measure molecular interactions [4] as well as mechanical tension 21,28, 43, the field of muscle tissue research is partially 
hampered in exploiting these tools due in part to the limited access to high end fluorescence microscopy. In recent years, the study of skeletal muscle development and disease is shifting from animal models to new in vitro muscle tissue approaches, that promise more controlled experimental approaches with improved optical access. Furthermore, these engineered muscle tissues not only avoid ethical considerations, but also overcome several problems of animal models such as a high price, time consuming procedures, and in some cases, failure to accurately predict human treatment response due to species specific differences 12, 18, 34. Although 2D skeletal muscle cell cultures can provide ease of predictions during drug testing and disease modelling [50], these systems are of limited use in contraction studies due to randomly oriented myotubes and the inability to maintain long-term cultures $14,22,39,49]$. In contrast, modern reconstituted $3 \mathrm{D}$ in vitro skeletal muscle systems are demonstrated to be an efficient tool for rapid and reliable drug screening [2, 54 and allow for testing of personalized treatments on cells harvested from individual patients. Besides these medical advantages, such functional muscle tissues were reported to successfully mimic native muscle tissue with long-term structural integrity [23 27] and allow new insights and fundamental knowledge of muscle tissue development, force generation during contraction as well as the phases of disease onset and progression [2, $11,19,24,25,31,32,41,48,51$. 51 . In culture platforms allowing for in situ force measurements, 3D skeletal muscle tissues self organize between mm sized posts around which muscle precursor cells are seeded together with an extracellular matrix. These chambers are commonly based on polydimethylsiloxane (PDMS) molds that can be tuned in their elastic properties and are soft enough to allow measurement of muscle contraction forces by recording the post deflection. Such non-invasive measurements of force generation in reconstituted muscles are indeed based on the elastic properties of PDMS 1. 29. A downside of PDMS is that the poor optical properties paired with routinely used material thickness prohibits high numerical aperture objectives that are necessary for high resolution microscopy, and hence make modern 3D microscopy methods like confocal and spinning disk microscopy on living tissue impossible. Instead, to study 3D tissues with high resolution requires fixation and removal from the culture device, thus preventing research on questions related to dynamic processes. Hence, they are of only limited use for the investigation of spatiotemporal research questions such as dynamics of cell-cell interaction or myoblast fusion. Moreover, PDMS has an immense capacity to absorb chemicals and proteins and is therefore unsuitable for serum-free medium applications or precise drug evaluation [52].

Recently, a method to determine contraction forces of myotubes grown within collagen or other ECM-like matrices using 3D traction force microscopy was described 42. This approach resolves force generation not only at the endpoints of the myotubes, as in the post systems, but can also determine traction forces along the length of the myotube. Although this new method dramatically enhances the resolution of force generation and is compatible with high resolution microscopy, it is limited to forces acting on the interface between the muscle cells and the provided environment. The force and tension distribution within the tissue, and namely between individual cells, remains only accessible by locally cutting myotubes, and thus damaging the system.

While the local distribution of tension in reconstituted muscle tissues cannot be measured at the moment, mechanical tension and its distribution in other tissues was previously shown to guide many fundamental biological processes such as collective cell migration, tissue morphogenesis and cell fate decisions [15, 45, 57. Despite this, non-destructive experimental approaches to investigate spatial and temporal forces on a cellular level are limited and thus, characterization of local cell niches within a tissue remains a challenge. To overcome this problem, Campas et al. introduced 
biocompatible oil microdroplets to evaluate cell-generated forces in living tissue for the first time [10] and very recently, deformable PAA beads were used to determine local tension on a cell scale within cancer spheroids, zebrafish embryos and during phagocytosis $13,28,53,55]$. Contrary to oil microdroplets, PAA beads are compressible and are therefore able to reveal isotropic tissue pressure.

In this study, we open new ways to study the global and local mechanical properties of biomimetic skeletal muscle tissue. We first present a novel technique to culture in vitro skeletal muscle tissues in $3 \mathrm{D}$ that inverses the geometry, so that the tissue is located closely to a microscopy glass slide, which allows real-time high resolution imaging, while still self organizing around thin posts of polymethylmethacrylate (PMMA) that are connected to the top of the chamber and thereby allowing both, accurate quantification of global forces via post deflection and high resolution 3D fluorescence microscopy. Next, we incorporate custom-made elastic PAA beads into the biomimetic muscle tissues to trace local tension within the tissues during development. both, the global and local forces match in magnitude, as expected. Interestingly, we observe an immense increase in local tension inside muscle tissues in vitro within the first week of development which remains at a similar level in the following week, although myotube diameter continues to increase. Notably, global muscle tissue pre-tension did not increase in the second week of differentiation, either. By enabling real-time high resolution microscopy on 3D in vitro muscle tissues for the first time, we envision our novel reconstituted muscle tissue device will be an advantage to the skeletal muscle research community by enabling the dynamic study of (sub-)cellular events.

\section{Materials and Methods}

\section{PMMA mold fabrication for 3D muscle tissue culture}

Muscle tissue culture molds consists of two main parts, both milled from polymethyl methacrylate (PMMA) as depicted in figure 11A. The bottom part of the PMMA chamber contains the ellipsoid culture wells (diameters: $3.5 \mathrm{~mm}, 6.5 \mathrm{~mm}$ ) and is glued onto a microscopy cover glass (VWR, Radnor, USA) using PDMS (Sylgard® 170 silicone, Sigma, St. Louis, USA) which is cured for $24 \mathrm{~h}$ at room temperature. The upper part represents the PMMA lid, which extends two $16 \mathrm{~mm}$ long ellipsoid posts per well (diameters: $0.68 \mathrm{~mm}, 1.3 \mathrm{~mm}$ ) with a distance of $3.3 \mathrm{~mm}$ between each opposing posts. Prior to use, the molds were sterilized using $70 \%$ ethanol and the wells were coated with a Poloxamere solution in $\mathrm{ddH}_{2} \mathrm{O}$ over night at $4{ }^{\circ} \mathrm{C}(5 \%$ Pluronic $\mathrm{R}$ F-127, Sigma, St. Louis, USA) to render the surface non-adhesive. MyoTACTIC PDMS molds were fabricated exactly as described in [1].

\section{$2 \mathrm{D}$ and $3 \mathrm{D}$ skeletal muscle progenitor culture}

The $\mathrm{C} 2 \mathrm{C} 12$ mouse muscle progenitor cell line was obtained from ATCC. The AB1190 and AB1167 immortalized human muscle progenitor cell line 33] was obtained from Vincent Mouly (Paris, France). Channelrhodopsin-2 was stably introduced into the AB1190 cell line by lentiviral transduction as described on the 8 webpage. The Fubi-ChR2-GFP plasmid was a gift from Edward Boyden (Addgene plasmid \#22051) 7. LifeAct-GFP and H2B-mCherry was stably introduced into the AB1167 cell line by lentiviral transduction. The pLenti6-H2B-mCherry plasmid was a gift from Torsten Wittmann (Addgene plasmid \#89766) 37 and the pLenti.PGK.Lifeact-GFP.W plasmid was a gift from Rusty Lansford (Addgene plasmid \#51010). Following lentiviral infection and a period of culture to expand the population, the cell populations were sorted to enrich for transduced cells. $\mathrm{C} 2 \mathrm{C} 12$ cells were cultivated in tissue culture flasks 
(75 $\mathrm{cm}^{2}$, Greiner, Kremsmünster, Austria) in $20 \mathrm{ml}$ Dulbecco's Modified Eagle Medium (DMEM, Capricorn, Ebsdorfergrund, Germany) containing $10 \%$ fetal calf serum (FCS, Sigma, St. Louis, USA) and $1 \%$ penicillin-streptomycin (Gibco, Waltham, USA) at 37 ${ }^{\circ} \mathrm{C}, 5 \% \mathrm{CO}_{2}$ in a humidified incubator. For cultivation of AB1190 and AB1167 cells, cultivation conditions were similar to those for $\mathrm{C} 2 \mathrm{C} 12$, with the exception that Skeletal Muscle Cell Growth medium (PROMOCELL, Heidelberg, Germany) was used as the base medium instead of DMEM.

$3 \mathrm{D}$ skeletal muscle tissues were raised in culture as previously reported $[2,31]$. Briefly, $1.5 \times 10^{7}$ cells per ml were resuspended in an ECM mixture containing DMEM $(40 \% \mathrm{v} / \mathrm{v}), 4 \mathrm{mg} / \mathrm{ml}$ bovine fibrinogen (Sigma, St. Louis, USA) in $0.9 \%(\mathrm{w} / \mathrm{v}) \mathrm{NaCl}$ solution in water and Geltrex ${ }^{T M}(20 \% \mathrm{v} / \mathrm{v}$, Gibco, Waltham, USA). Custom-made fluorescent PAA beads were directly added to the mixture (1000 beads per ml of ECM mixture). $25 \mu \mathrm{l}$ of the cell mixture was utilized for each tissue in the PMMA molds and $15 \mu \mathrm{l}$ for the MyoTACTIC PDMS molds 1. Fibrin polymerisation was induced with thrombin (Sigma, St. Louis, USA) at 0.5 units per $\mathrm{mg}$ of fibrinogen for $5 \mathrm{~min}$ at $37^{\circ} \mathrm{C}$. Subsequently, $300 \mathrm{\mu l}$ growth medium consisting of DMEM supplemented with $20 \%$ FCS, $1 \%$ penicillin-streptomycin and $1.5 \mathrm{mg} / \mathrm{ml} 6$-aminocaproic acid (ACA, Sigma, St. Louis, USA) was added. After 2 days, the growth medium was exchanged to differentiation medium consisting of DMEM supplemented with $2 \%$ horse serum (HS, Sigma, St. Louis, USA), $1 \%$ penicillin-streptomycin (Gibco, Waltham, USA) and $2 \mathrm{mg} / \mathrm{ml} \mathrm{ACA.}$ For human microtissues the differentiation medium was additionally supplemented with $10 \mathrm{\mu g} / \mathrm{ml}$ insulin. The differentiation medium was changed every other day.

\section{PAA bead fabrication}

To produce PAA beads a water-in-oil emulsion approach was used. Since in this study predominantly $2.1 \mathrm{kPa}$ (Young's modulus) beads were utilized, the fabrication and characterization for this elasticity is described, representatively. To generate beads with other elasticity, adapted concentrations of monomers and crosslinkers are required.

The water phase was prepared by mixing two parts of acrylamide solution $(40 \% \mathrm{v} / \mathrm{v}$, Sigma, St. Louis, USA) with one part of N,N'-methylenebisacrylamide solution (2\% $\mathrm{v} / \mathrm{v}$, Sigma, St. Louis, USA). This PAA solution was then diluted to $13.5 \%$ (v/v) using a $65 \%(\mathrm{v} / \mathrm{v})$ phosphate buffered saline (PBS, Sigma, St. Louis, USA) to obtain a pre-bead-mix. The mechanical properties of the PAA beads can be tuned by changing the dilution of the pre-bead-mix. The oil phase was composed of $3 \%$ Span®80 (Sigma, St. Louis, USA) in n-hexane (Merck, Darmstadt, Germany). Shortly before polymerization the pre-bead-mix as well as the oil phase were degassed for 10 min at 50 mbar. The free-radical cross-linking polymerization of the PAA solution was initiated by adding $1.5 \%$ (w/v) ammonium persulphate (APS, AppliChem, Darmstadt, Germany) and the $\mathrm{pH}$-value of the solution was neutralized using $\mathrm{NaOH}$ solution. The emulsion was then generated by injecting the pre-bead-mix into the n-hexane with a $100 \mathrm{\mu l}$ Hamilton syringe (Hamilton, Reno, USA). Hereafter, the polymerization was catalysed by adding $3 \%$ (v/v) N,N,N',N'-tetramethylethylenediamine (TEMED, Sigma, St. Louis, USA) and the emulsion was degassed again for $6 \mathrm{~min}$. The supernatant was discarded and the polymerization was kept at $85^{\circ} \mathrm{C}$ for 10 min. To reach end of gelation the beads were incubated at room temperature over night. Next day, the beads were washed five times with n-hexane and transferred to $65 \%$ PBS. Finally, the beads were labeled fluorescently with ATTO-565-NHS-ester solution (Atto-Tech, New York, USA). For that purpose, the labeling solution was incubated with the beads for at least $30 \mathrm{~min}$ at room temperature and washed three times with $65 \%$ PBS afterwards. The fluorescent intensity of the beads was increased by repeating the labeling process three to five times. 


\section{Mechanical characterization of PAA beads}

To characterize the mechanical properties of the elastic PAA beads the shear modulus $G$ and bulk modulus $K$ were determined. The shear modulus was measured via active microrheology using a custom optical tweezers setup. For this purpose, smaller beads with a diameter of $1 \mu \mathrm{m}$ (Latex beads, Sigma, St. Louis, USA) were embedded into the PAA beads. The complex shear modulus, $G^{*}=G^{\prime}+i G^{\prime \prime}$, was measured for varying oscillation-frequency of the $1 \mu \mathrm{m}$ beads [3]. The shear modulus $G$ used for further analysis was estimated by averaging shear storage moduli $G^{\prime}$ for low frequencies from 1 $\mathrm{Hz}$ to $10 \mathrm{~Hz}$. The bulk modulus $K$ was measured via an osmotic pressure approach using 2 MDa dextran molecules (Dextran T2000, Pharmacosmos, Wiesbaden, Germany) which were dissolved in the PAA beads solution. Since the pore size of the PAA gel is much smaller than the hydrodynamic radius of the dextran, an osmotic pressure $p=K \frac{\Delta V}{V}$ acts on the PAA beads and gives rise to a compression $\Delta V / V$ proportional to the bulk modulus $K$. The exerted osmotic pressure depends on the concentration of dextran and has been calibrated previously 35]. Here, three different concentrations $(60$ $\mathrm{g} / \mathrm{l}, 85 \mathrm{~g} / \mathrm{l}$ and $100 \mathrm{~g} / \mathrm{l}$ ) were used, corresponding to osmotic pressures of $6 \mathrm{kPa}, 12 \mathrm{kPa}$ and $18 \mathrm{kPa}$, respectively. The distribution of PAA bead diameters in the three solutions was measured and compared to the uncompressed situation by segmenting images of PAA beads with the find-edges-function of Fiji and fitting ellipses to the edges. The bulk modulus $K$ was determined by a linear fit of the volume change $\Delta V / V$ to osmotic pressure $p$.

\section{Bead deformation analysis (BDA)}

In order to reconstruct forces acting in the tissue, the deformation of embedded PAA beads were quantified using a two step process implemented in a graphical user interface developed in Matlab (Mathworks, US). First, the beads were segmented and fitted using spherical harmonics. Then an analytical solution to the elastic problem was applied to gain the forces on the beads. For bead segmentation, a small volume around each PAA bead was cut out of the 3D image stack and if desired the effective pixel density was interpolated by a factor of 2 for display purposes. In the cutout a threshold intensity was determined according to segmentation parameters that were typically set to clipping level $(50 \%)$ and peak intensity voxels $(3 \%)$ and the image was then transformed into a binary image. Subsequently, the surface was determined by extracting voxels at the edge of the binary volume and the algorithm returns the coordinates $\left(x_{i}, y_{i}, z_{i}\right)$ of these voxels that do form the surface of the PAA bead. In order to quantitatively capture the deformation, spherical harmonics were fitted to the segmented bead surface using a Nelder-Mead simplex algorithm. As we were only interested in the dominant forces acting on the bead, we took advantage of the mode decomposition in spherical harmonics and restricted the fit to spherical harmonics $Y_{n m}(\theta, \phi)$ of degree $n=0,2$ and order $m=0$. While the $Y_{00}$ mode gives the radius of the sphere, the $Y_{20}$ mode is the mode of dominant compression, where the z-direction was determined by rotating the sphere until the absolute value of the $Y_{20}$ mode became maximal. This ansatz focuses on the direction of major deformation of the PAA bead, and it furthermore uses the angular symmetry around the z-axis that is implied in the order $m=0$.

In detail, following this approach, the coordinates of the segmented surface $\left(x_{i}, y_{i}, z_{i}\right)$ were transformed into spherical coordinates $r(\theta, \phi)$. The surface defined by the spherical harmonics

$$
r_{\mathrm{sh}}(\theta)=\sum_{n=0,2} c_{n 0} Y_{n 0}(\theta, \phi)=\frac{c_{00}}{\sqrt{4 \pi}}+c_{20} \sqrt{\frac{5}{16 \pi}} \cdot\left(3 \cos ^{2} \theta-1\right)
$$


was then fitted to the segmented surface by rotating $r_{\mathrm{sh}}(\theta, \phi)$ to align with the major direction of deformation of $r(\theta, \phi)$ and minimizing the residual $\left(r_{\mathrm{sh}}-r\right)^{2}$ by varying $c_{00}$ and $c_{20}$. Thus, a sphere of radius $c_{00} / \sqrt{4 \pi}$ with uniaxial deformation proportional to $c_{20}$ was fitted to the deformed PAA bead. This fitting procedure was repeated five times with randomly picked starting values in each case. The parameter combination with the lowest residual was taken as the result. The sought force dipole $\vec{F}$ acting on the PAA bead was finally calculated by

$$
\begin{gathered}
\vec{F}=\frac{G}{2} c_{00} c_{20} \sqrt{5}\left(\frac{N_{\mathrm{r}}(\nu)}{4}+\frac{3 N_{\theta}(\nu)}{2}\right) \vec{e}_{\mathrm{z}} \\
N_{\mathrm{r}}(\nu)=\frac{2 \nu}{1-2 \nu}+\frac{1}{2(2-3 \nu)(1-2 \nu)}+\frac{1}{2(2-3 \nu)} \\
N_{\theta}(\nu)=\frac{1}{2}-\frac{1}{2(2-3 \nu)}
\end{gathered}
$$

with the scaling factors $N_{r}$ and $N_{\theta}$ as well as $\vec{e}_{\mathrm{z}}$ being the unit vector on the main deformation axis. Equation 2 was derived following classical elasticity theory [30], resulting in expression for the stress on the sphere that was then integrated over the half sphere to get the force dipole acting on the PAA bead. The axis of the force dipole relates to the axis of rotation when fitting the spherical harmonics.

\section{Immunostaining and confocal fluorescence microscopy}

3D skeletal muscle tissues were washed once with PBS and fixed in $4 \%$ paraformaldehyde (PFA) for $15 \mathrm{~min}$ at room temperature in the culture device and afterwards washed three times with PBS, again. Frozen tissues were sectioned, mounted as $12 \mu \mathrm{m}$ sections and rehydrated. Next, the tissues were blocked for $1 \mathrm{~h}$ at room temperature using PBS supplemented with $20 \%$ goat serum (GS, Sigma, St. Louis, USA) and $0.2 \%$ Triton-X-100 (Carl Roth, Kalsruhe, Germany). Subsequently, the samples were incubated with the primary antibody (monoclonal mouse anti-sarcomeric alpha actinin, 1:100, Abcam, Cambridge, UK) diluted in blocking solution over night at $4{ }^{\circ} \mathrm{C}$. After three washes with blocking solution, the samples were incubated with the appropriate secondary antibody (polyclonal goat anti-mouse IgG H\&L, 1:1000, Abcam, Cambridge, UK) diluted in blocking solution for $45 \mathrm{~min}$ at room temperature. The cell nuclei were counterstained using Hoechst 33342 (1:1000, ThermoFisher, Waltham, USA). Confocal images were acquired using Slidebook 6 software (3i, Denver, USA) using an inverted microscope (Nikon Eclipse Ti-E, Minato, Japan) equipped with a CSU-W1 spinning disk head (Yokogawa, Musashino, Japan) and a scientific CMOS camera (Prime BSI, Photometrics, Tucson, USA). Images were analysed and prepared for publication using the open source software Fiji 47 .

\section{Multiview imaging}

Fixed muscle tissues were mounted in fluorethylenpropylene (FEP) capillaries (Proliquid, Überlingen, Germany) together with $1 \%$ low-melting-agarose solution (LMA, Invitrogen, Carlsbad, USA) for multiview imaging using a Z1 Light-Sheet microscope (Zeiss, Oberkochen, Germany). The LMA solution contained fluorescent sub-resolution beads (100 nm diameter, Invitrogen, Carlsbad, USA) that were used for registration of the images acquired from different views. The samples were imaged from at least four different views with angles in the range 60 to 90 between the views. Afterwards, the images from different views were registered and fused using the Multiview Deconvolution software [40]. The program detects the positions of the registration beads and overlays the images accordingly. The registration beads were also 
used to estimate the point spread function (PSF) of the microscope. Knowing the PSF, the images were deconvolved and fused.

251

\section{Post deflection analysis}

For post deflection analysis, we focused the top part of the post with the imaging system and recorded a time series. Thanks to the high resolution microscopy compatibility of the new chamber design, the outline of the post showed a strong contrast to the surrounding tissue. Utilizing a custom written program in Matlab (Mathworks, Natick, USA), a line of pixels located at the center of the post of each image was chosen for every time point. By calculating the gradient of pixel intensities along this line, the outline of the post appeared as a strong peak in the signal. Subpixel resolution was achieved by the fit of a Gaussian function to these peaks and determining the central position. Subsequently, the pixel values were converted into units of length using the pre-calibrated pixel sizes. Analysis of the entire time stack in that manner gave rise to a time dependent displacement signal for the post edges. Finally, the pulling forces were determined by multiplication of the post displacement by an apparent spring constant of the post. This apparent spring constant was estimated by finite element analysis (Inventor, Autodesk, San Rafael, USA) considering the post's exact geometry and material properties. The assumption of a linear force to displacement relation is valid for the observed displacements of less than $45 \mu \mathrm{m}$.

\section{Relation between directionality of tension}

As crosscheck between the global (post based) and local (bead based) tension we can estimate the expected local tension using the post deflection measurements. First, we determine the tensional stress in the tissue along the post-post axis by dividing the global force $f_{t}=0.3 \pm 0.1 \mathrm{mN}$ by the tissue diameter $A=0.17 \pm 0.03 \mathrm{~mm}^{2}$, yielding a tensional stress of $t=1.8 \pm 0.67 \mathrm{kPa}$. To compare this tension, which acts along the length of the tissue (x-axis) with the deformation which is typically along $\mathrm{y}$ and $\mathrm{z}$, a simple calculation shows that in isotropic materials of Poisson ratio $\nu=0.5$ the absolute stress values are the same in all directions. This can be simply demonstrated by recalling Hooke's law in 3D and considering a constraint deformation, where only a deformation in $\mathrm{x}$ is considered and $\mathrm{y}, \mathrm{z}$ are assumed to be fixed. Hooke's law gives

$$
\begin{gathered}
\sigma_{x x}=\frac{E}{(1+\nu)(1-2 \nu)}\left((1-\nu) u_{x x}+\nu\left(u_{y y}+u_{z z}\right)\right) \\
\sigma_{y y}=\frac{E}{(1+\nu)(1-2 \nu)}\left((1-\nu) u_{y y}+\nu\left(u_{x x}+u_{z z}\right)\right) \\
\sigma_{z z}=\frac{E}{(1+\nu)(1-2 \nu)}\left((1-\nu) u_{z z}+\nu\left(u_{x x}+u_{y y}\right)\right) .
\end{gathered}
$$

The constraint deformation enforces that only the component $u_{x x}$ is different from zero, hence

$$
\begin{aligned}
\sigma_{x x} & =\frac{E(1-\nu)}{(1+\nu)(1-2 \nu)} u_{x x} \\
\sigma_{y y} & =\sigma_{z z}=\frac{\nu E}{(1+\nu)(1-2 \nu)} u_{x x}
\end{aligned}
$$

which means that the stresses are not independent, but that

$$
\sigma_{y y}=\sigma_{x x} \frac{\nu}{1-\nu} \text {. }
$$

In first order, the tissue around the bead remains mostly undeformed for the small deformations measured, hence the absolute stress $\sigma_{y y}$ acts on the beads. For a Poisson ratio $\nu=0.5$ the tensional stress in $\mathrm{x}$ direction is equal in magnitude to the measured stress in $\mathrm{y}$ and $\mathrm{z}$ direction.

Therefore we can directly compare the measured local tension derived by the bead deformation of $2.4 \pm 0.9 \mathrm{kPa}$ to the global tension of $1.8 \pm 0.67 \mathrm{kPa}$, and confirm that these match within the error.

\section{3}

254

255

256

257

258

259

260

261

262

263

264

265

266

267 


\section{Statistical analysis}

All results are presented as mean \pm SD and statistical differences of experimental groups were analysed by unpaired t-test using GraphPad Prism 6.0 software, where $\mathrm{p}<$ 0.05 was considered as significant. Significances were subdivided into three levels: $\quad(p=$ $0.05-0.01),(\mathrm{p}=0.01-0.001), \quad(\mathrm{p}<0.001)$ The number of biomimetic muscle tissues $(\mathrm{N})$ and analysed PAA beads (n) is indicated in every figure legend. In Figure $4 \mathrm{E}$ 'n' refers to the number of measured myotubes.

\section{Code availability}

The bead deformation analysis as well as the post deflection open source software is available on GitHub: 36,58 .

\section{Results}

\section{In vitro development of functional $3 \mathrm{D}$ skeletal muscle tissues compatible with high resolution video microscopy}

The combination of high resolution microscopy and reconstituted three-dimensional (3D) skeletal muscle microtissues holds the potential to lend new insights into the formation and maturation of skeletal muscle cells. To enable such studies, we re-envisioned the design of currently used 3D muscle microtissue culture platforms, which led to the establishment of a two part chamber system. The bottom component is milled from a PMMA block to contain eight individual oval-shaped cell culture chambers (Figure 1A, bottom; Supplementary Figure 1). This bottom part is glued to a standard microscopy coverslip, which then enables high resolution inverted fluorescence microscopy compatible with oil or water immersion objectives. The upper part is also machined from PMMA and fits tightly into the bottom part. It consists of long vertical posts (Figure 1A, top; Supplementary Figure 1) around which the 3D muscle tissue anchors during formation. When the two halves of the culture device are placed together, the vertical posts nearly touch the bottom, thus confining the muscle tissue to a region close to the bottom of the coverslip. A hole in the top part positioned equidistant between each pair of posts allows for gas and medium exchange during growth and measurement (Supplementary Figure 1).

Biomimetic 3D muscle tissues arise from a self-organization process when an initial mixture of mononucleated skeletal muscle progenitors (aka 'myoblasts') in a Geltrex@-Fibrin matrix is seeded into the bottom of each device well (Figure 1 $\mathrm{B}$ ), similar to the tissue formation in previously described PDMS-based culture systems [1,2, 31. To demonstrate the imaging quality and observation capacity of this chamber design, $\mathrm{C} 2 \mathrm{C} 12$ mouse myoblasts were seeded within a Geltrex@-Fibrin matrix at defined ratios and cultured in growth medium for two days allowing for equilibration to the $3 \mathrm{D}$ environment. The medium was then exchanged to a low serum formulation to support differentiation (i.e. fusion to form multinucleated 'myotubes') for a period of up to 2 weeks (Figure 1 C). During remodeling, the tissues anchored to each end of the posts, which act as artificial tendons (Figure 1B). Resulting tissues fixed and immunostained for sarcomeric alpha actinin (SAA), and counterstained using Hoechst 33342 to visualize nuclei, revealed multinucleated myotubes aligned in parallel between the posts, as well as striations indicative of sarcomere structures characteristic of myotubes progressing through the process of maturation (Figure 1D). These in-plate observations of sarcomere structures required high resolution fluorescence microscopy, which is not possible in the context of a PDMS-based cultivation system, when imaging through the PDMS. To further characterize biomimetic muscle tissue development, 

myotubes which are consistently aligned close to each other and are evenly distributed throughout the entirety of the tissue (Figure $1 \mathrm{E}$ ).

To demonstrate the capacity of time-lapse high resolution microscopy on living 3D skeletal muscle tissues, we generated a Lifeact-GFP and H2B-mCherry labeled human myoblast cell line (AB1167-Lifeact-GFP-H2B-mCherry) and recorded a timeseries of 10 day old 3D skeletal muscle tissue dynamics using a spinning disk microscope. The results show that although the tissue appears immobile at the macroscopic level, or in snapshots, a continuous rearrangement at the cell level takes place (Supplementary Video 1). On a subcellular level we observe highly dynamic nuclear motion during myotube progressing maturation (Figure $1 \mathrm{~F}$, Supplementary Video 2). Hence, our approach enables the formation of 3D biomimetic muscle tissues with characteristic skeletal muscle features that are in close proximity to a glass window, thus enabling high resolution and time resolved 3D microscopy, which represents a highly advanced alternative to previously described culture systems.

\section{Global contraction forces of in vitro skeletal muscle tissues}

The most common approach to determine the contractile forces of a reconstituted muscle tissue is to monitor the deflection of elastic PDMS posts and to then calculate contractile forces using the spring constant of the posts [1. Indeed, an advantage of PDMS is that the elasticity, and hence the spring constant can be tuned by the degree of crosslinking during curing. Although the elasticity of the PMMA material used to construct our device cannot be easily changed, variations in the posts' geometry provide the possibility of controlling the spring constant. Namely, the diameter and the length of the posts are sensitive parameters responsible for their compliance. The spring constant of $16 \mathrm{~mm}$ long PMMA posts used in our system is $39 \mathrm{\mu N} / \mu \mathrm{m}$, which is considerably higher than commonly used PDMS posts. However, this disadvantage is compensated by the highly improved imaging capacity, that allows for determining deflection amplitudes down to $0.2 \mu \mathrm{m}$, which corresponds to a force resolution of \pm 7.8 $\mu \mathrm{N}$. To test if the global tissue contractile force detection is comparable to that reported for human skeletal muscle tissue formed in PDMS-based systems, 2 week old skeletal muscle tissues generated from an immortalized human myoblast line 20 genetically modified to stably express the light-sensitive channelrhodopsin-2 ion channel 7 (AB1190-Fubi-ChR2-GFP) were investigated in our chamber system.

Consistent with prior work, we find a clear deflection of the posts upon comparing the position of the posts when the muscle tissue is relaxed versus in a contracted situation [46. For both optogenetically and acetylcholine (ACh) triggered contractions, we measured deflections that were several micrometers (Figure 2A, Supplementary Video $3+4$ ). Owing to high contrast imaging of the PMMA posts made feasible by the culture device, it is possible to precisely trace the post deflection and determine the contractile forces. While optogenetically induced twitch contractions exhibited contractile forces of $0.2 \pm 0.04 \mathrm{mN}$ on average, the tetanus contractions induced by treatment with $2 \mathrm{mM}$ of acetylcholine elicited contractile forces of about $1.1 \pm 0.3 \mathrm{mN}$ (Figure 2 B). These measurements are consistent with force measurements obtained in PDMS based chambers [1]. Our post deflection analysis software is able to trace exerted forces on the post over time from acquired videos with minimal background signal of \pm $8.5 \mu \mathrm{N}$ (Figure $2 \mathrm{C}$ ). For optogenetic twitches we observe a roughly one second contraction phase before the in vitro muscle tissue returned to the relaxed state. By comparison, a tetanus contraction induced by $2 \mathrm{mM}$ ACh reached its peak of contractile force more slowly and the time elapsed before return to the relaxed state was almost 25 seconds (Figure 2D).

We further established a method to evaluate pre-tension during skeletal muscle 
A

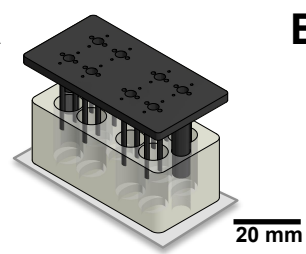

C $20 \%$

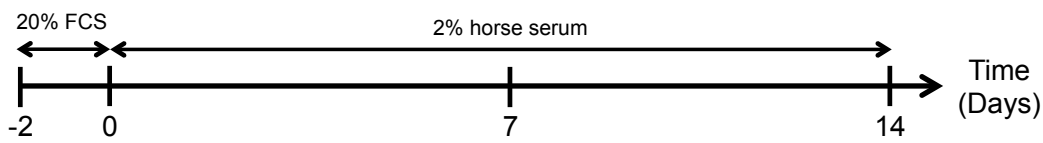

D
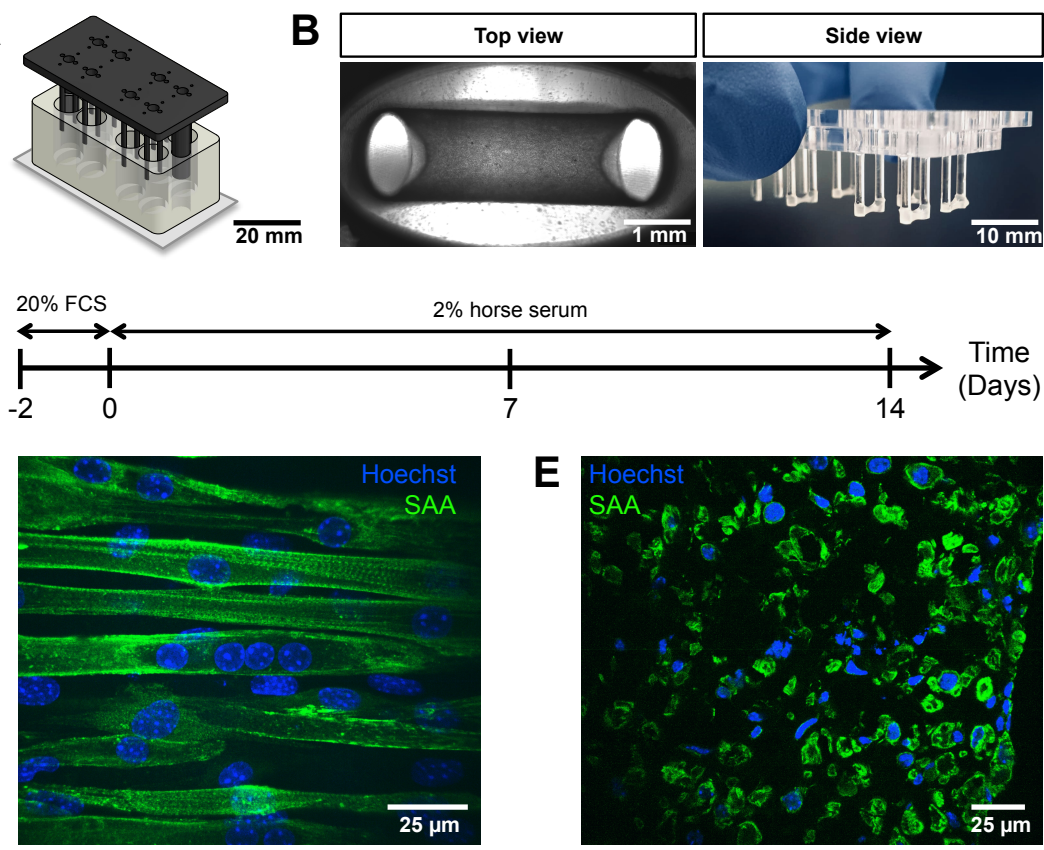

E

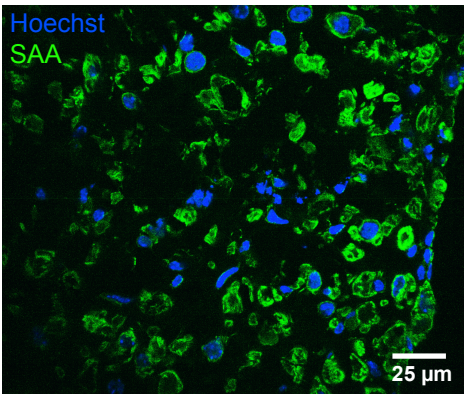

$\mathbf{F}$
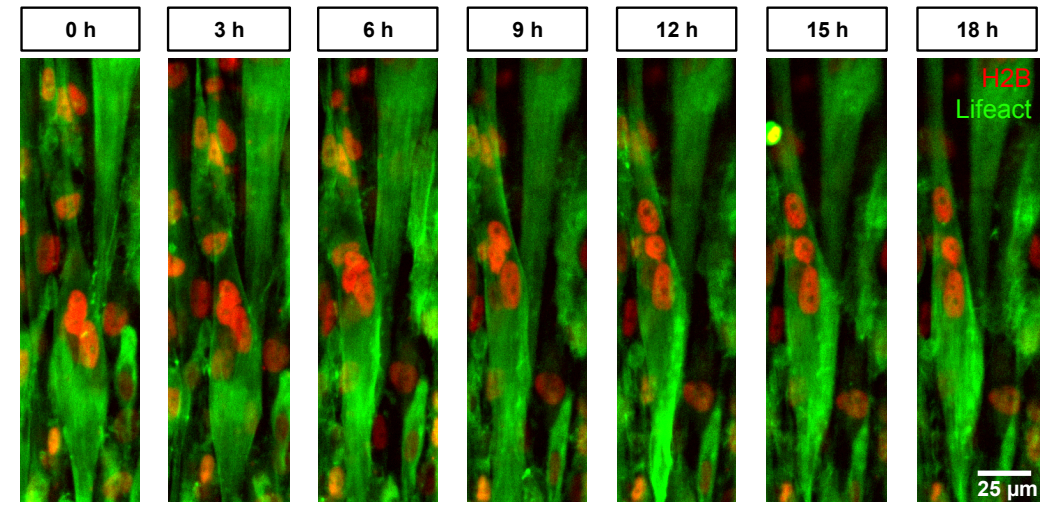

Figure 1. PMMA culture device supports the generation of $3 \mathrm{D}$ biomimetic skeletal muscle tissues. A, Computer generated depiction of the PMMA mold design. The top part containing eight pairs of vertical posts is shown in black. Eight holes positioned equidistant between each pair of posts allows for gas and media exchange. Two larger sized vertical posts at either end of the top part serve to fix the top and bottom portion (translucent white) together and ensure vertical posts are properly positioned. The bottom portion is affixed to a microscopy grade glass. B, Images of remodeled $\mathrm{C} 2 \mathrm{C} 12$ muscle tissues at 7 days of differentiation anchored to the end of the posts captured to provide a view looking up into a well in which the top and bottom parts are fashioned together (left; 4X objective) or from the side looking at the top part to visualize 6 pairs of posts with tissues at the bottom. C, Schematic workflow used to raise 3D skeletal muscle tissues in vitro. D-E, Representative confocal microscopy longitudinal (D, whole mount, flattened stack, 40X water immersion objective) and transverse (E, cryosection, single snap, 40X water immersion objective) images of multinucleated myotubes within a 14 day old muscle tissue immunostained for sacomeric alpha-actinin (SAA, green) and counterstained with Hoechst 33342 to visualize nuclei (blue). The cross section of the tissue was measured to be $0.17 \pm 0.03 \mathrm{~mm}^{2}$. F, Timeseries of nuclear motion withing developing 3D skeletal muscle tissue 10 days after differentiation, demonstrating the possibility of high resolution imaging during living tissue formation. Lifeact-GFP (green) and H2B-mCherry (red) was stably introduced into AB1167 cells. 
tissue development in vitro. Specifically, we treated C2C12 skeletal muscle tissues with $10 \%$ SDS to dissolve the myotube membranes, thereby releasing the pre-tension established by the myotubes, which is visualized by the PMMA posts returning to their original position upon dissolution of the muscle tissue (Supplementary Video 5). Utilizing our post deflection analysis software (accessible on GitHub, 36 ) we quantified the pre-tension to be $0.3 \pm 0.1 \mathrm{mN}$ for skeletal muscle tissues cultured for a period of one week (Figure 2E), and this did not change significantly in the subsequent week of culture. Taken together, we demonstrate that the culture device allows for determination of both contractile forces as well as tissue pre-tension simply via post deflection analysis.
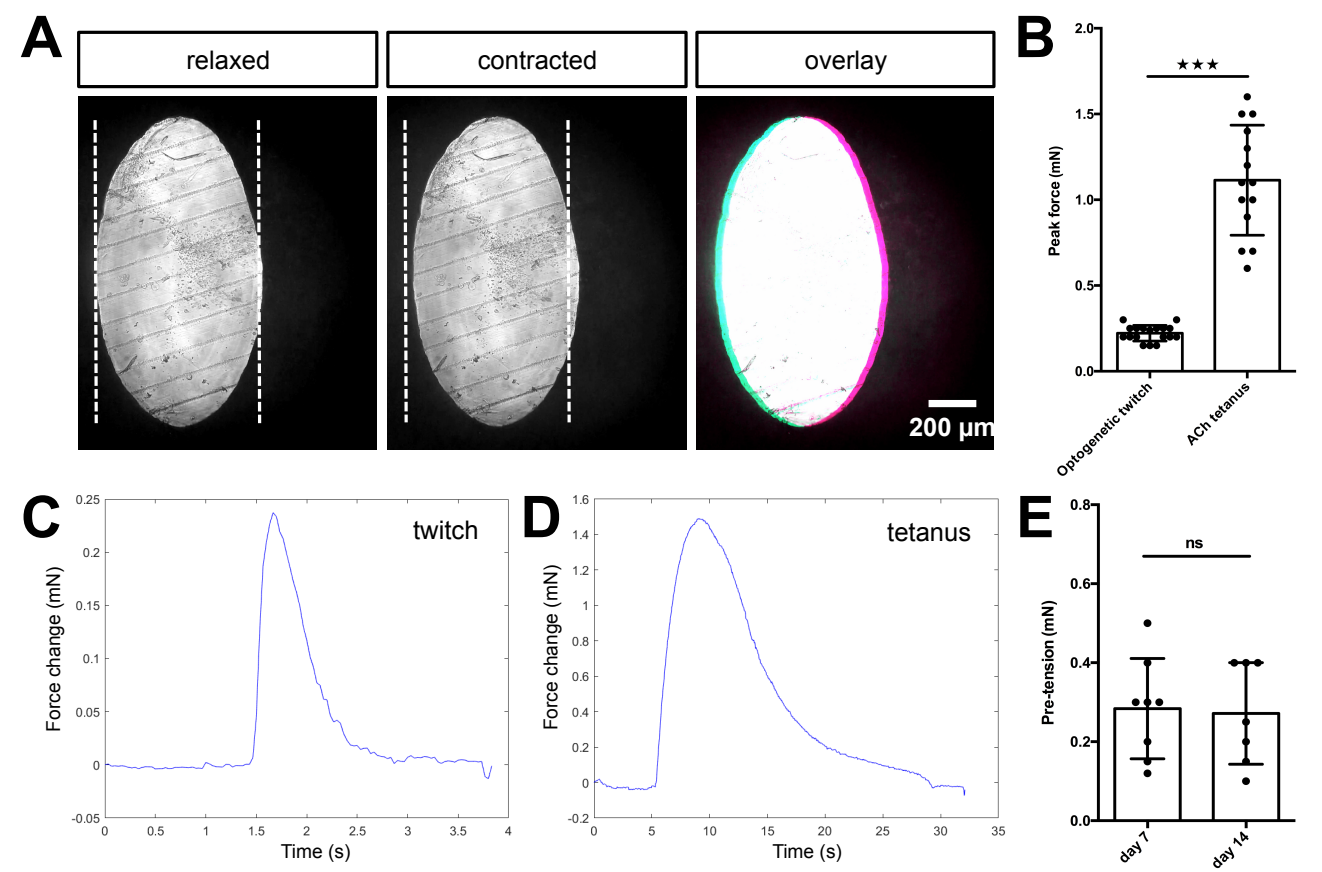

Figure 2. Quantification of biomimetic skeletal muscle tissues contractile forces over time in culture. A, Representative bright field images of the bottom of a post under 10x magnification before (left) and during (middle) a tetanus contraction. Pink and blue pseudo color highlights the deflection regions when pre- and post- contraction images are overlaid (right). B, Bar graph with force measurements of optogenetically induced twitches $(\mathrm{N}=18)$ and ACh tetanus contractions $(\mathrm{N}=14)$ of two week old muscle tissues. Representative time course traces of forces exerted on the posts is displayed in $\mathbf{C}$ for an optogenetically induced twitch and in $\mathbf{D}$ for a tetanus contraction induced by the addition of $2 \mathrm{mM}$ ACh. E, Bar graph with pre-tension measurements of skeletal muscle tissues at one $(\mathrm{N}=8)$ and two weeks of differentiation $(\mathrm{N}=7)$.

\section{Characterization and computational analysis of elastic PAA beads}

To determine the local tension in the muscle tissue, we implemented elastic hydrogel beads as tension sensors, an approach first used by others in different systems 13, 28,53,55]. We custom-made elastic PAA beads by a water in oil emulsion approach (Figure 3A). The use of PAA beads as tension sensors requires precise mechanical characterization. Using optical tweezer based active microrheology on $1 \mu \mathrm{m}$

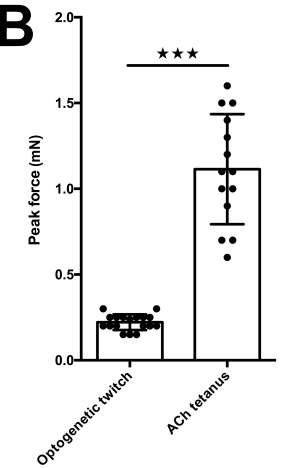


polystyrene particles embedded within the PAA beads, we determined a shear modulus of $G=710 \pm 270 \mathrm{~Pa}$ by averaging shear storage moduli $G^{\prime}$ for low frequencies from $1 \mathrm{~Hz}$ to $10 \mathrm{~Hz}$ (Figure $3 \mathrm{~B}$ ). Additionally, the bulk modulus was determined to be $K=41 \pm 6$ $\mathrm{kPa}$ by an osmotic pressure approach (Figure $3 \mathrm{C}$ ). Here three different concentrations of $2 \mathrm{MDa}$ dextran $(60 \mathrm{~g} / \mathrm{l}, 85 \mathrm{~g} / \mathrm{l}$ and $100 \mathrm{~g} / \mathrm{l})$ were applied to the elastic beads, which corresponds to osmotic pressures of $6 \mathrm{kPa}, 12 \mathrm{kPa}$ and $18 \mathrm{kPa}$, respectively. PAA bead diameter in the three solutions was measured and compared to the corresponding uncompressed situation. Consequently, the Poisson ratio $\nu=(3 K-2 G) /(6 K+2 G)$ was determined as $\nu=0.491 \pm 0.005$, which is consistent with previous reports of Poisson's ratio in PAA 5. For the force calculation, the Young's modulus $E=2 G(1+\nu)$ of the PAA beads used in this study was hence determined to be $E=2.1 \pm 0.8 \mathrm{kPa}$.

We next used the mechanical properties of the PAA beads to determine the forces exerted on the beads, from images where bead deformation was measured. Previous studies by others used finite element methods for the analysis [53], which have the disadvantage of being computationally time consuming. Here, we focused on an analytical solution that yields the dominant tension dipole, and the spatial orientation of this dipole force. The advantage of this approach is the high speed of analysis, and the reduction of the relevant forces into a simple scalar force number, while reporting also the direction of force tension propagation in the tissue. We performed the analysis using a custom written program that implements an automated computational bead deformation analysis (BDA), which is capable of deconvolving microscope images using a given point spread function (PSF), extracting the edges of beads, and finally fitting spherical harmonics to the segmented beads to register the bead deformation (Figure 3D). Finally, BDA uses the identified coefficients of the spherical harmonic fit to analytically calculate the dominant force acting on the PAA bead. The BDA software has an user friendly interface (Figure $3 \mathrm{E}$ ) and is available on GitHub 58.

\section{Local tension within in vitro skeletal muscle tissues during development}

We then conducted a quantitative study of local tension within 3D skeletal muscle microtissues, which was made possible by integrating PAA beads within tissues and conducing high-resolution microscopy in our custom culture platform. Specifically, 3D skeletal muscle tissues were raised from $\mathrm{C} 2 \mathrm{C} 12$ cells in the PMMA based chambers in which PAA beads were included in the starting cell-matrix suspension to produce microtissues with PAA beads stably embedded throughout. The tissues containing the PAA beads were then monitored and the tension was determined within the developing tissues over 2 weeks. Immunostaining of 14 days old tissues revealed normal in vitro muscle tissue development featuring multinucleated myotubes aligned parallel to each other with typical sarcomere structures (Figure 4A). Images of muscle tissue cross sections revealed dense biomimetic muscle tissues with PAA beads stably incorporated and spread evenly throughout the whole tissue between the myotubes (Figure 4B). These observations suggest that the presence of elastic beads does not disturb the development of the muscle tissues.

We first imaged the PAA beads contained within the living muscle microtissues via spinning disc microscopy on the same day we seeded the $\mathrm{C} 2 \mathrm{C} 12$ cells mixed within the ECM matrix into the culture device. The culture chamber is crucial to obtain fluorescence images of the beads that have sufficient quality for subsequent BDA. We repeated this tension investigation in living tissues at 7 and 14 days of differentiation, respectively. Whereas the beads were fairly spherical directly after seeding, we observed significantly higher deformation, and therefore tension, within the tissues after one week of differentiation (Figure $4 \mathrm{C}$ ). The average internal tissue tension at this time-point was 
A

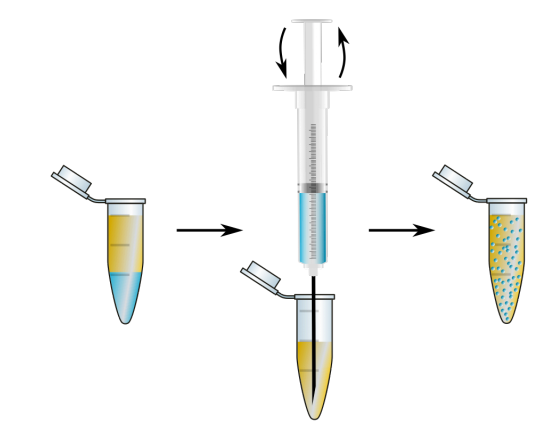

C

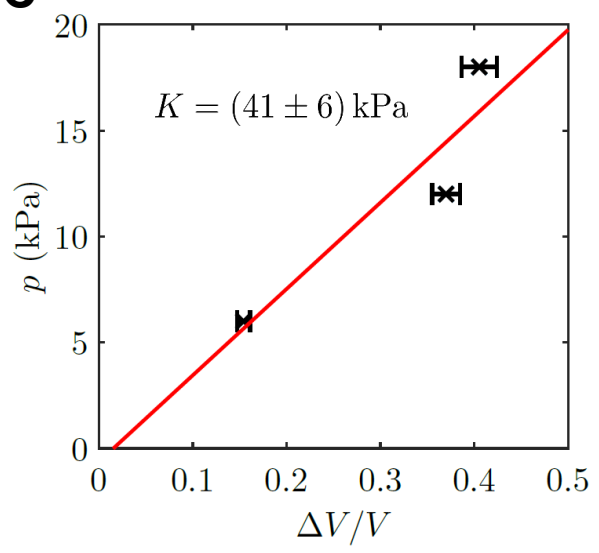

B

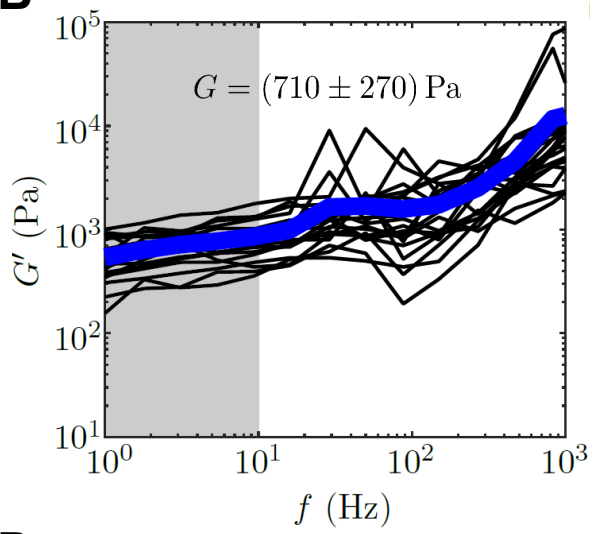

D

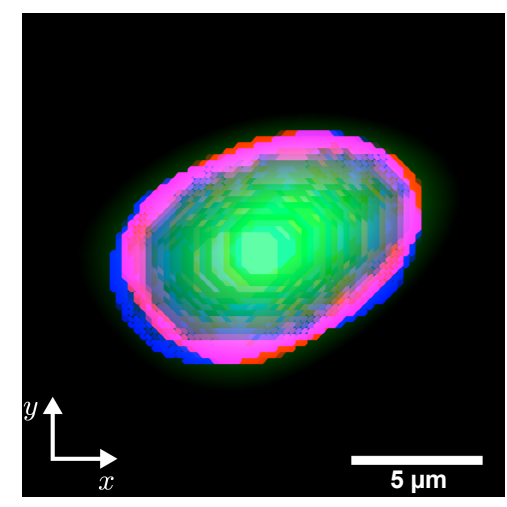

E

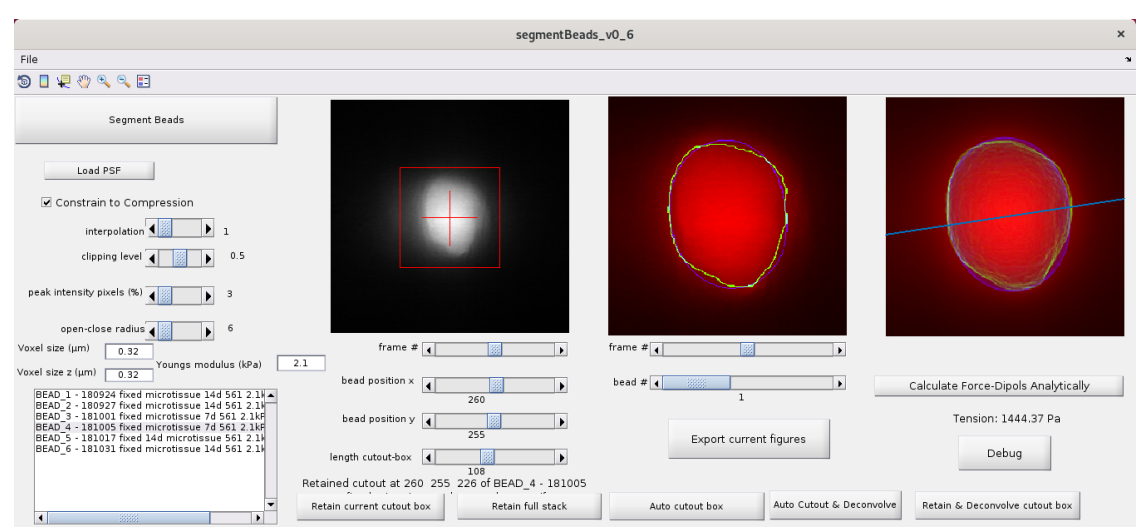

Figure 3. PAA bead characterization and analysis software. A, Schematic workflow of bead fabrication. Using a syringe a water in oil emulsion is created and the acryl amide solution is polymerized to produce elastic PAA beads. B, Graph showing the determination of the shear modulus of elastic PAA beads, which was measured for varying oscillation-frequency of $1 \mu \mathrm{m}$ beads. $\mathbf{C}$, Graph depicting the determination of the bulk modulus of elastic PAA beads, which was measured by an osmotic pressure approach using dextran. D, Segmentation of a deformed PAA bead. The surface (red) was segmented and a linear combination of spherical harmonics of degree $\mathrm{n}=$ 0,2 and order $m=0$ (blue) were fitted to the surface. E, Screenshot of PAA bead deformation analysis software front-end. Providing a point spread function enables the automatized program to deconvolve the image and determine the edge position of an elastic bead (green) in a given cutout box. The bead's shape is then fitted by spherical harmonics, which are used to calculate the main axis of the force dipole (blue line) and the corresponding tension analytically. 
slightly more than $2.4 \pm 0.9 \mathrm{kPa}$, and maintained a similar level of tension following 2 weeks of differentiation. As a control experiment, we measured the tension of beads embedded in ECM matrix only made of geltrex and fibrin, and without cells. We found no significant difference between beads in ECM matrix without cells after seven days when compared to beads measured directly after seeding. This demonstrates that the observed bead deformation is indeed due to remodeling and differentiation of the cells in the ECM matrix. To further control these measurements, we cultivated 3D muscle tissues with very stiff $150 \mathrm{kPa}$ beads integrated throughout. At this high stiffness, we expect that the measured forces cannot deform the beads sufficiently for detection with microscopy. Indeed, in this case we did not observe deformation of the PAA beads after one week of differentiation as compared to the day of tissue seeding (Supplementary Figure 2). Consistently, when 7 days old tissues with PAA beads embedded are dissolved using 5\% SDS, the beads return to their initial spherical shape (Figure $4 \mathrm{C}$ ).

To further validate our results acquired via spinning disk microscopy in PMMA/glass molds, we produced $\mathrm{C} 2 \mathrm{C} 12$-derived muscle microtissues in the previously described MyoTACTIC PDMS mold [1] and imaged fixed tissues that were removed from the mold and imaged in a FEP capillary using Multiview Light-Sheet microscopy to provide a comparison. Multiview Light-Sheet microscopy has the advantage of isotropic resolution, and hence checks potential problems due to spinning disk microscopy and deconvolution. We first conducted studies and confirmed that fixation of in vitro skeletal muscle tissues per se did not impair embedded PAA bead shape significantly by comparing images of the same bead before and after fixation in our PMMA/glass molds (Supplementary Figure 3). Consistent with bead data obtained using spinning disk confocal microscopy in our PMMA/glass device, we observed a significant increase of local tension exerted on the elastic beads after 7 days of skeletal muscle microtissue differentiation of slightly more than $2.3 \pm 0.9 \mathrm{kPa}$ (Figure 4D), but again, no significant difference was detected after a second week of differentiation. Importantly, myotube diameter increased significantly from $10.5 \pm 1.4 \mu \mathrm{m}$ at one week of differentiation to $13.6 \pm 2.5 \mu \mathrm{m}$ in the second week (Figure $4 \mathrm{E}$ ). This shows that while the initial phase of tissue compaction myotube differentiation influences beads tension, a further increase in myotube diameter does not result in more tension on embedded PAA beads.

\section{Discussion}

The emergent importance of mechanobiology has demonstrated that mechanical interaction between cells as well as stiffness and tensile forces provide important signalling elements for cell biology and cell fate. Muscle tissue is intrinsically exposed to large forces and rapid changes in tension and stiffness. Hence, it is to be expected that the mechanical properties of the environment are of particular relevance for the homeostasis of muscle tissue. To study these interactions, not only the precise observation of self organized $3 \mathrm{D}$ tissue is required, but also a non-invasive readout of global and local forces is important.

Furthermore, in vitro skeletal muscle tissue model systems provide an enormous potential to give new insights into muscle development, degradation, repair and dynamics. However, the usability of these novel systems has been limited by several key weaknesses of the currently used culturing methods. While the PDMS based elastomers used in such post based chambers is well studied and under control, its optical properties prevent the usage of high numerical aperture objectives and hence high resolution fluorescence microscopy. Furthermore, PDMS acts like a protein sponge that absorbs large amounts of proteins from the medium, thus preventing the use of special serum free media that are required in modern stem cell approaches. Here we solve both problems by using an inverted geometry chamber that is made of PMMA. The material 
A

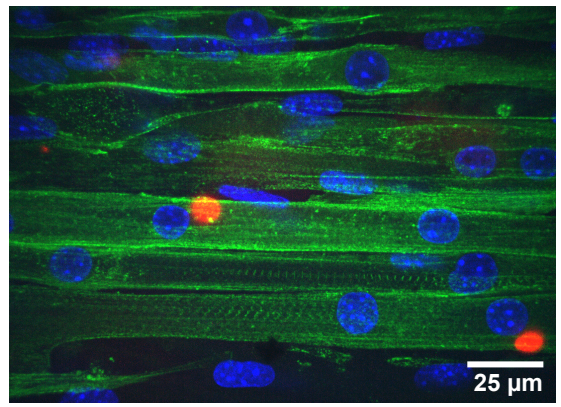

C

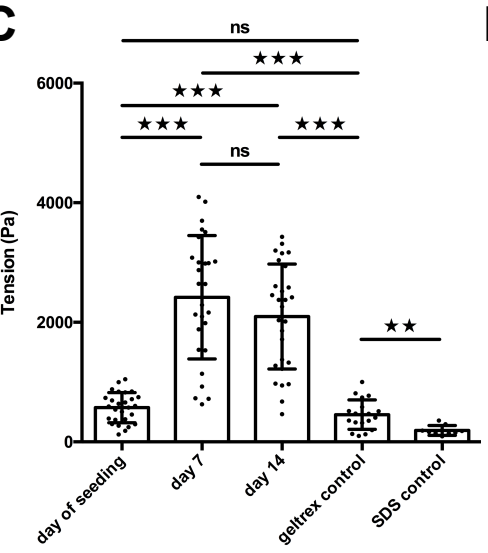

D
B
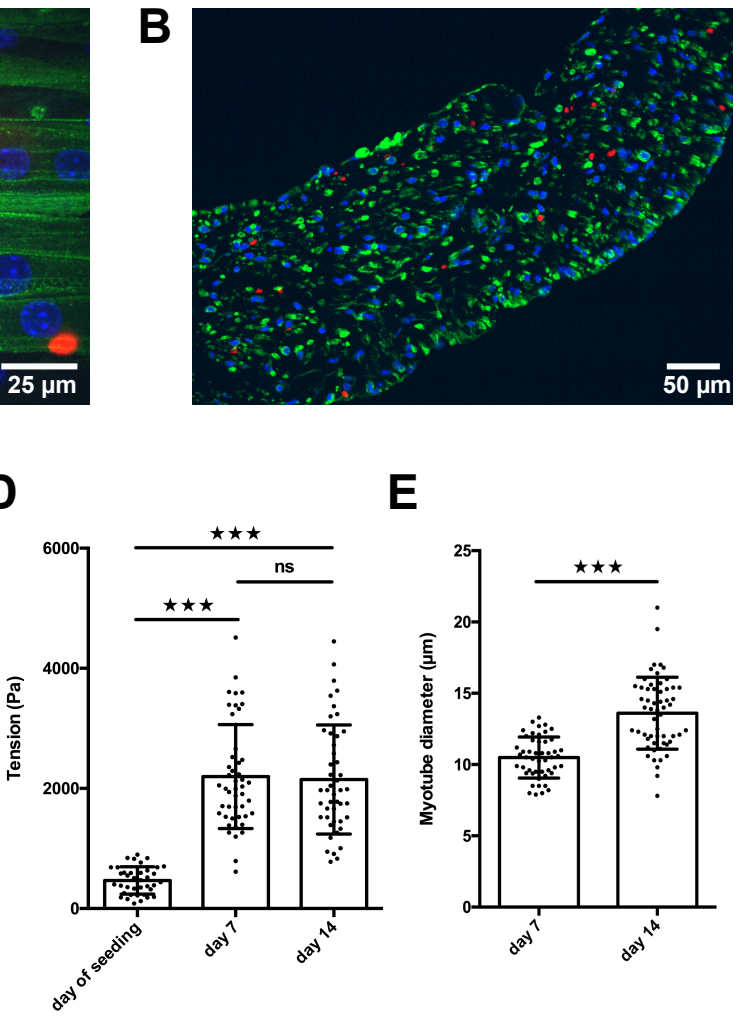

$\mathbf{E}$

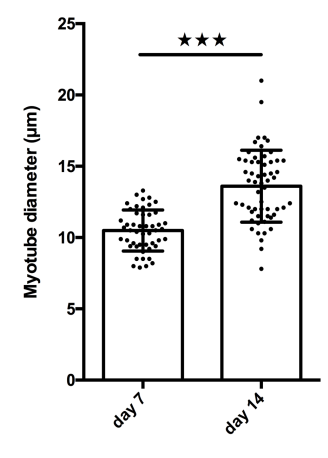

Figure 4. PAA beads serve as local tension sensors within in vitro muscle tissues. A, Representative flattened confocal microscopy stack of a 14 day old C2C12 tissue with PAA beads embedded (red) and immunostained for sacomeric alpha-actinin (green) and counterstained with Hoechst 33342 to visualize nuclei (blue). B, Representative confocal snap of a transverse section of a 14 day old $\mathrm{C} 2 \mathrm{C} 12$ tissue. Color coding is identically equal to A. C, Bar graph of local tension within $\mathrm{C} 2 \mathrm{C} 12$ muscle tissues at the day of seeding $(\mathrm{N}=7, \mathrm{n}=28)$, and after one $(\mathrm{N}=4, \mathrm{n}=28)$ and two weeks of differentiation $(\mathrm{N}=4, \mathrm{n}=29)$. Beads were imaged in the context of living tissues raised in PMMA molds for BDA utilizing spinning disk microscopy. Also shown is negative controls in which tension was measured in the cell-free geltrex/fibrin matrix alone $(\mathrm{N}=3$, $\mathrm{n}=20)$ as well as following the dissolution of tissues using $5 \%$ SDS $(\mathrm{N}=3, \mathrm{n}=10) . \mathbf{D}$, Bar graph of local tension within $\mathrm{C} 2 \mathrm{C} 12$ muscle tissues raised in the MyoTACTIC PDMS microtissue platform that were fixed and removed for imaging using Multiview Light-Sheet microscopy on the day of seeding $(\mathrm{N}=13, \mathrm{n}=39)$, and after one $(\mathrm{N}=17, \mathrm{n}=48)$ and two weeks of differentiation $(\mathrm{N}=19, \mathrm{n}=46)$. $\mathbf{E}$, Bar graph of myotube diameter analysis conducted on $\mathrm{C} 2 \mathrm{C} 12$ muscle tissues at one $(\mathrm{N}=3, \mathrm{n}=49)$ and two $(\mathrm{N}=3, \mathrm{n}=57)$ weeks of differentiation. Each myotube diameter data point reflects the average of three measurements per myotube. 
prevents the protein sponge effect, while the geometry allows positioning a glass coverslip in close proximity to the self-organized muscle tissue, thus enabling high resolution microscopy.

Changing the material and adapting the geometry to still enable global force measurements while gaining high quality optical access is a key advancement. It now allows time resolved high resolution measurements of in vitro muscle tissue development, myotube formation and fusion, without the need of fixation and tissue removal as necessary in the previous designs. Using fluorescent protein tagging it was directly possible to gain high resolution 3D images of actin networks with a high time resolution. Such fluorescent protein based life stains in combination with this approach will help understanding the dynamic interactions between muscle cells during differentiation, fusion, myotube formation and maturation. Therefore, we enable real-time high resolution imaging during cultivation of in vitro muscle tissues for the first time which is of great benefit for various future research issues such as myoblast fusion or myotube maturation. A previous 2.5D myotube culture approach was indeed able to monitor first myotube maturation and nuclear movement to the periphery of skeletal myotubes by dynamic high resolution imaging [44]. However, this approach cannot draw conclusions within a $3 \mathrm{D}$ system, is not capable to conduct functional or contraction studies and is furthermore unable to quantify force generation of the tissue, which all can be combined using our novel approach.

The here presented 3D in vitro muscle tissues raised close to a glass coverslip displayed myotube structures characteristic for progressing maturation e.g. sacomere striations and multinucleation (Figure 1) and indeed showed functional responses to contractile stimuli (Figure 2 Supplementary Video 3). While the force measurement via post deflection is a commonly used method 29], we now offer an advanced and reliable readout for global force generation which is even more precise due to sharply milled post edges and higher quality of imaging through glass. We therefore pave the way for future contraction studies of diseased or individual patient related tissues. As for instance, by shortening the height of the PMMA posts we can vary the spring constant of the posts dramatically and therefore enable first isometric contraction investigations of skeletal muscle tissues in vitro which is impossible using highly flexible PDMS.

To enable the study of potential correlations of contractile forces and tension between cells, we exploit the post deformations to determine the overall tissue contractility while the deformable beads are used to measure the local tension between individual cells and tubes. PAA beads were just previously reported to function as tension sensors within cancer spheroids in vitro, during phagocytosis as well as within zebrafish embryos in vivo $13,28,53,55]$. Combining local and global analysis is only possible with the new chamber design, as the bead deformation analysis relies on high resolution images. Furthermore, in contrast to the previous published tension analysis programs, the here introduced approach focuses on the principal components of tissue tension and directionality, which largely simplifies the analysis and the comparison between different treatments, cell types and contraction trigger approaches. The access to the global and the local tension also allows to independently crosscheck the values. Assuming a Poisson ratio of 0.5 and using the measured global tensional force as determined by the post deflection of $f_{t}=0.3 \pm 0.1 \mathrm{mN}$ (Figure 2) as well as the cross section area of $A=0.17 \pm 0.03 \mathrm{~mm}^{2}$ (Figure 1) of the in vitro skeletal muscle tissue, we use $t=f_{t} / A$ to predict a local average tension of $t=1.8 \pm 0.67 \mathrm{kPa}$, which is in excellent agreement to the average value of $2.4 \pm 0.9 \mathrm{kPa}$ obtained by the elastic bead analysis (Figure 4, see Methods and Material for details). This crosscheck suggests that the post deflection measurements are already sufficient to determine average tension in the tissue, but the local measurement can be used to then test the tension as function of position in the tissue. 
Establishing the combined approach for the first time in developing 3D skeletal muscle tissues in vitro, we observe a significant increase in local tension between myotubes in $\mathrm{C} 2 \mathrm{C} 12$ cells after one week of differentiation which does not further increase in the following week (Figure 4). Interestingly, we do not observe an increase of global tissue pre-tension in the second week of differentiation, either (Figure 2). However, we indeed monitor a significant increase in myotube diameter from week one to week two of differentiation as others reported before 1, 2, 31. In addition, Afshar et al. showed slight in vitro muscle tissue remodelling from week one to week two of differentiation. Hence, neither myotube diameter nor progressing tissue remodelling has a significant impact on local cellular tension within the 3D muscle tissues. Therefore, a mechanical homeostasis may be reached on a cellular level among myoblast fusion, myotube death and myotube progressing maturation after one week of in vitro muscle tissue differentiation. Furthermore, we can speculate that myotubes may be protected by a mechanical buffer layer composed of e.g. large glycocalyx biopolymers that are known to have crucial mechanical functions for cells and cell aggregates [17]. Our results suggest that cellular tension within in vitro muscle tissues is more connected to tissue pre-tension.

The novel culture mold is milled using PMMA and hence, easy and reliable to fabricate. The chamber design also allows scaling to a large number of experiments in parallel, which is a key element for screening approaches. It was designed for sufficient gas exchange, easy medium exchange as well as drug delivery via holes in the lid of the mold. Utilizing PMMA and microscopy glass for our molds, we overcome the issues of immense chemical absorption by the material and poor optical properties that previous approaches possessed 1, 2, 31. Thus, we can additionally offer serum-free culturing of in vitro skeletal muscle tissues and accurate drug dose response studies.

In conclusion, we provide a novel technique for functional 3D in vitro skeletal muscle tissue cultivation that enables real-time high resolution microscopy of living 3D biomimetic muscle tissue for the first time. We use the new approach for first global as well as local cellular force investigations of developing in vitro muscle tissues. We observe that cellular tension in $\mathrm{C} 2 \mathrm{C} 12$ in vitro muscle tissues is closely related to global tissue pre-tension and reaches a mechanical homeostatic phase after one week of differentiation. However, in vitro skeletal muscle tissue maturation is still progressing in the second week of differentiation, interestingly. Further, cultivation of muscle tissues directly on glass enables whole new opportunities to study highly complex and dynamic issues of myogenesis in $3 \mathrm{D}$ in vitro. In addition, an easy and reliable readout of contractile forces makes the novel culture mold applicable for individualised drug screening as well as diagnosis.

\section{Supporting Information}

\section{Author contribution}

Author 1, Author 8 and Author 9 designed the research. Author 1 carried out most experiments and Author 2 performed Light-Sheet and control experiments. Author 3 programmed the post deformation software and analysed respective data. Author 4 and 5 programmed the Bead deformation analysis software, which was used by Author 1 for respective data analysis. Author 4 also contributed to bead fabrication and characterisation. Author 6 and Author 7 provided training to Author 1 in muscle progenitor 2D and 3D culture and analysis. Author 7 created the stable cell lines. Author 6 fabricated MyoTACTIC PDMS plates. Author1, Author 8, and Author 9 wrote the article. 


\section{Supplementary Figure 1}

Engineering drawing with respective dimensions of the novel culture device used in this study for raising $3 \mathrm{D}$ skeletal muscle tissues in vitro.

\section{Supplementary Figure 2}

Stiff $150 \mathrm{kPA}$ PAA beads do not get significantly deformed during biomimetic muscle tissue development. $\mathrm{C} 2 \mathrm{C} 12$ microtissues with $2.1 \mathrm{kPa}$ embedded were cultivated for 2 weeks and the PAA bead deformations were captured at the day of seeding $(\mathrm{N}=13, \mathrm{n}=39)$ and after 7 days $(\mathrm{N}=17, \mathrm{n}=48)$ as well as 14 days of differentiation $(\mathrm{N}=19, \mathrm{n}=46)$, respectively. In parallel $\mathrm{C} 2 \mathrm{C} 12$ microtissues were cultivated with $150 \mathrm{kPa}$ beads embedded. $150 \mathrm{kPa}$ beads were not significantly more deformed after 7 days of differentiation $(\mathrm{N}=4, \mathrm{n}=21)$ in comparison to $2.1 \mathrm{kPa}$ beads at the day of seeding.

\section{Supplementary Figure 3}

Stiff 150 kPA PAA beads do not get significantly deformed during biomimetic muscle tissue development. $2.1 \mathrm{kPa}$ PAA beads were embedded in $\mathrm{C} 2 \mathrm{C} 12$ microtissues and cultivated for one week. The exact same PAA beads were imaged and its deformation was analysed before and after fixation, respectively.

\section{Supplementary Video 1}

$3 \mathrm{D}$ rendering of a $19 \mathrm{~h}$ timeseries of a developing human microtissue 10 days after differentiation shows continuous cellular rearrangement. Lifeact-GFP (white) and H2B-mCherry (red) was stably introduced into AB1167 cells.

\section{Supplementary Video 2}

Flattened $18 \mathrm{~h}$ timeseries stack of a developing human microtissue 10 days after differentiation shows highly dynamic nuclear motion during myotube progressing maturation. Lifeact-GFP (green) and H2B-mCherry (red) was stably introduced into AB1167 cells.

\section{Supplementary Video 3}

2 week old human microtissue contracts upon optogenetically induced stimulus. Channelrhodopsin-2 was stably introduced into AB1190 cells.

\section{Supplementary Video 4}

Post deflection upon contraction of a 2 week old human AB1190 microtissue induced by $2 \mathrm{mM} \mathrm{ACh}$.

\section{Supplementary Video 5}

Post deflection during dissolution of a 1 week old human AB1167 microtissue with $10 \%$ SDS. 


\section{Acknowledgments}

This work was funded by the Human Frontiers Science Program (to PMG and TB). MEA received funding from the Natural Sciences and Engineering Research Council Training Program in Organ-on-a-Chip Engineering and Entrepreneurship Scholarship. PMG is the Canada Research Chair in Endogenous Repair. Funding to PMG is from the Natural Sciences and Engineering Research Council and Medicine by Design, a Canada First Research Excellence Program. TB was supported by the European Research Council (consolidator grant number 771201). We thank Yannik Vaas for his excellent technical expertise during mold fabrication.

\section{References}

1. M. E. Afshar, H. Y. Abraha, M. A. Bakooshli, S. Davoudi, N. Thavandiran, K. Tung, H. Ahn, H. J. Ginsberg, P. W. Zandstra, and P. M. Gilbert. A 96-well culture platform enables longitudinal analyses of engineered human skeletal muscle microtissue strength. Sci Rep, 10(1):6918, Apr. 2020.

2. M. Afshar Bakooshli, E. S. Lippmann, B. Mulcahy, N. Iyer, C. T. Nguyen, K. Tung, B. A. Stewart, H. van den Dorpel, T. Fuehrmann, M. Shoichet, A. Bigot, E. Pegoraro, H. Ahn, H. Ginsberg, M. Zhen, R. S. Ashton, and P. M. Gilbert. A 3D culture model of innervated human skeletal muscle enables studies of the adult neuromuscular junction. Elife, 8, May 2019.

3. W. W. Ahmed, Fodor, M. Almonacid, M. Bussonnier, M.-H. Verlhac, N. Gov, P. Visco, F. van Wijland, and T. Betz. Active Mechanics Reveal Molecular-Scale Force Kinetics in Living Oocytes. Biophys. J., 114(7):1667-1679, 2018.

4. W. R. Algar, N. Hildebrandt, S. S. Vogel, and I. L. Medintz. FRET as a biomolecular research tool - understanding its potential while avoiding pitfalls. Nat. Methods, 16(9):815-829, 2019.

5. T. Boudou, J. Ohayon, C. Picart, and P. Tracqui. An extended relationship for the characterization of Young's modulus and Poisson's ratio of tunable polyacrylamide gels. Biorheology, 43(6):721-728, 2006.

6. D. Bourgeois, A. Regis-Faro, and V. Adam. Photoactivated structural dynamics of fluorescent proteins. Biochem. Soc. Trans., 40(3):531-538, June 2012.

7. E. S. Boyden, F. Zhang, E. Bamberg, G. Nagel, and K. Deisseroth. Millisecond-timescale, genetically targeted optical control of neural activity. Nat. Neurosci., 8(9):1263-1268, Sept. 2005.

8. Broad-Institute. Lentiviral transduction protocols, Jan. 2020.

9. M. Buckingham. Skeletal muscle formation in vertebrates. Current Opinion in Genetics $\&$ Development, 11(4):440-448, Aug. 2001.

10. O. Campàs, T. Mammoto, S. Hasso, R. A. Sperling, D. O'Connell, A. G. Bischof, R. Maas, D. A. Weitz, L. Mahadevan, and D. E. Ingber. Quantifying cell-generated mechanical forces within living embryonic tissues. Nat. Methods, 11(2):183-189, Feb. 2014.

11. A. J. Capel, R. P. Rimington, J. W. Fleming, D. J. Player, L. A. Baker, M. C. Turner, J. M. Jones, N. R. W. Martin, R. A. Ferguson, V. C. Mudera, and M. P. Lewis. Scalable 3D Printed Molds for Human Tissue Engineered Skeletal Muscle. Front Bioeng Biotechnol, 7:20, 2019. 
12. J. A. DiMasi, R. W. Hansen, and H. G. Grabowski. The price of innovation: new estimates of drug development costs. J Health Econ, 22(2):151-185, Mar. 2003.

13. M. E. Dolega, M. Delarue, F. Ingremeau, J. Prost, A. Delon, and G. Cappello. Cell-like pressure sensors reveal increase of mechanical stress towards the core of multicellular spheroids under compression. Nat Commun, 8:14056, 2017.

14. D. Eberli, S. Soker, A. Atala, and J. J. Yoo. Optimization of human skeletal muscle precursor cell culture and myofiber formation in vitro. Methods, 47(2):98-103, Feb. 2009.

15. A. J. Engler, S. Sen, H. L. Sweeney, and D. E. Discher. Matrix elasticity directs stem cell lineage specification. Cell, 126(4):677-689, Aug. 2006.

16. L. Fenno, O. Yizhar, and K. Deisseroth. The development and application of optogenetics. Annu. Rev. Neurosci., 34:389-412, 2011.

17. J. G. Gandhi, D. L. Koch, and M. J. Paszek. Equilibrium Modeling of the Mechanics and Structure of the Cancer Glycocalyx. Biophys. J., 116(4):694-708, 2019 .

18. F. P. Gaschen, E. P. Hoffman, J. R. Gorospe, E. W. Uhl, D. F. Senior, G. H. Cardinet, and L. K. Pearce. Dystrophin deficiency causes lethal muscle hypertrophy in cats. J. Neurol. Sci., 110(1-2):149-159, July 1992.

19. D. Gholobova, M. Gerard, L. Decroix, L. Desender, N. Callewaert, P. Annaert, and L. Thorrez. Human tissue-engineered skeletal muscle: a novel 3D in vitro model for drug disposition and toxicity after intramuscular injection. Sci Rep, 8(1):12206, 2018.

20. F. Girardi, A. Taleb, L. Giordani, B. Cadot, A. Datye, M. Ebrahimi, D. G. Gamage, D. P. Millay, P. M. Gilbert, and F. L. Grand. TGF signaling curbs cell fusion and muscle regeneration. preprint, Cell Biology, Feb. 2019.

21. C. Grashoff, B. D. Hoffman, M. D. Brenner, R. Zhou, M. Parsons, M. T. Yang, M. A. McLean, S. G. Sligar, C. S. Chen, T. Ha, and M. A. Schwartz. Measuring mechanical tension across vinculin reveals regulation of focal adhesion dynamics. Nature, 466(7303):263-266, July 2010.

22. X. Guo, K. Greene, N. Akanda, A. Smith, M. Stancescu, S. Lambert, H. Vandenburgh, and J. Hickman. In vitro Differentiation of Functional Human Skeletal Myotubes in a Defined System. Biomater Sci, 2(1):131-138, Jan. 2014.

23. M. Juhas, G. C. Engelmayr, A. N. Fontanella, G. M. Palmer, and N. Bursac. Biomimetic engineered muscle with capacity for vascular integration and functional maturation in vivo. Proc. Natl. Acad. Sci. U.S.A., 111(15):5508-5513, Apr. 2014.

24. J. H. Kim, I. Kim, Y.-J. Seol, I. K. Ko, J. J. Yoo, A. Atala, and S. J. Lee. Neural cell integration into 3D bioprinted skeletal muscle constructs accelerates restoration of muscle function. Nat Commun, 11(1):1025, Feb. 2020.

25. J. H. Kim, Y.-J. Seol, I. K. Ko, H.-W. Kang, Y. K. Lee, J. J. Yoo, A. Atala, and S. J. Lee. 3D Bioprinted Human Skeletal Muscle Constructs for Muscle Function Restoration. Sci Rep, 8(1):12307, 2018. 
26. F. Lauretani, C. R. Russo, S. Bandinelli, B. Bartali, C. Cavazzini, A. Di Iorio, A. M. Corsi, T. Rantanen, J. M. Guralnik, and L. Ferrucci. Age-associated changes in skeletal muscles and their effect on mobility: an operational diagnosis of sarcopenia. J. Appl. Physiol., 95(5):1851-1860, Nov. 2003.

27. P. H. U. Lee and H. H. Vandenburgh. Skeletal muscle atrophy in bioengineered skeletal muscle: a new model system. Tissue Eng Part A, 19(19-20):2147-2155, Oct. 2013.

28. W. Lee, N. Kalashnikov, S. Mok, R. Halaoui, E. Kuzmin, A. J. Putnam, S. Takayama, M. Park, L. McCaffrey, R. Zhao, R. L. Leask, and C. Moraes. Dispersible hydrogel force sensors reveal patterns of solid mechanical stress in multicellular spheroid cultures. Nat Commun, 10(1):144, 2019.

29. W. R. Legant, A. Pathak, M. T. Yang, V. S. Deshpande, R. M. McMeeking, and C. S. Chen. Microfabricated tissue gauges to measure and manipulate forces from 3D microtissues. Proceedings of the National Academy of Sciences, 106(25):10097-10102, June 2009.

30. A. I. Lurie and A. Belyaev. Theory of Elasticity. Springer-Verlag Berlin Heidelberg, Berlin, Heidelberg, 2005. OCLC: 699849378.

31. L. Madden, M. Juhas, W. E. Kraus, G. A. Truskey, and N. Bursac. Bioengineered human myobundles mimic clinical responses of skeletal muscle to drugs. Elife, 4:e04885, Jan. 2015.

32. S. M. Maffioletti, S. Sarcar, A. B. H. Henderson, I. Mannhardt, L. Pinton, L. A. Moyle, H. Steele-Stallard, O. Cappellari, K. E. Wells, G. Ferrari, J. S. Mitchell, G. E. Tyzack, V. N. Kotiadis, M. Khedr, M. Ragazzi, W. Wang, M. R. Duchen, R. Patani, P. S. Zammit, D. J. Wells, T. Eschenhagen, and F. S. Tedesco. Three-Dimensional Human iPSC-Derived Artificial Skeletal Muscles Model Muscular Dystrophies and Enable Multilineage Tissue Engineering. Cell Rep, 23(3):899-908, Apr. 2018.

33. K. Mamchaoui, C. Trollet, A. Bigot, E. Negroni, S. Chaouch, A. Wolff, P. K. Kandalla, S. Marie, J. Di Santo, J. L. St Guily, F. Muntoni, J. Kim, S. Philippi, S. Spuler, N. Levy, S. C. Blumen, T. Voit, W. E. Wright, A. Aamiri, G. Butler-Browne, and V. Mouly. Immortalized pathological human myoblasts: towards a universal tool for the study of neuromuscular disorders. Skelet Muscle, 1:34, Nov. 2011.

34. J. W. McGreevy, C. H. Hakim, M. A. McIntosh, and D. Duan. Animal models of Duchenne muscular dystrophy: from basic mechanisms to gene therapy. Dis Model Mech, 8(3):195-213, Mar. 2015.

35. S. Monnier, M. Delarue, B. Brunel, M. E. Dolega, A. Delon, and G. Cappello. Effect of an osmotic stress on multicellular aggregates. Methods, 94:114-119, Feb. 2016.

36. T. Muenker. Post Deflection Analysis. https://github.com/Tillmuen09/PostDeflectionAnalysis, May 2020.

37. H. Pemble, P. Kumar, J. van Haren, and T. Wittmann. GSK3-mediated CLASP2 phosphorylation modulates kinetochore dynamics. J. Cell. Sci., 130(8):1404-1412, 2017. 
38. M. Periasamy, S. K. Maurya, S. K. Sahoo, S. Singh, S. K. Sahoo, F. C. G. Reis, and N. C. Bal. Role of SERCA Pump in Muscle Thermogenesis and Metabolism. Compr Physiol, 7(3):879-890, 2017.

39. M. R. Pimentel, S. Falcone, B. Cadot, and E. R. Gomes. In Vitro Differentiation of Mature Myofibers for Live Imaging. J Vis Exp, (119), 2017.

40. S. Preibisch, F. Amat, E. Stamataki, M. Sarov, R. H. Singer, E. Myers, and P. Tomancak. Efficient Bayesian-based multiview deconvolution. Nat. Methods, 11(6):645-648, June 2014.

41. L. Rao, Y. Qian, A. Khodabukus, T. Ribar, and N. Bursac. Engineering human pluripotent stem cells into a functional skeletal muscle tissue. Nat Commun, 9(1):126, 2018.

42. M. Rausch, D. Böhringer, M. Steinmann, D. W. Schubert, S. Schrüfer, C. Mark, and B. Fabry. Measurement of Skeletal Muscle Fiber Contractility with High-Speed Traction Microscopy. Biophys. J., Dec. 2019.

43. P. Ringer, A. Weißl, A.-L. Cost, A. Freikamp, B. Sabass, A. Mehlich, M. Tramier, M. Rief, and C. Grashoff. Multiplexing molecular tension sensors reveals piconewton force gradient across talin-1. Nat. Methods, 14(11):1090-1096, Nov. 2017.

44. W. Roman, J. P. Martins, F. A. Carvalho, R. Voituriez, J. V. G. Abella, N. C. Santos, B. Cadot, M. Way, and E. R. Gomes. Myofibril contraction and crosslinking drive nuclear movement to the periphery of skeletal muscle. Nat. Cell Biol., 19(10):1189-1201, Oct. 2017.

45. K. Saha, A. J. Keung, E. F. Irwin, Y. Li, L. Little, D. V. Schaffer, and K. E. Healy. Substrate modulus directs neural stem cell behavior. Biophys. J., 95(9):4426-4438, Nov. 2008.

46. M. S. Sakar, D. Neal, T. Boudou, M. A. Borochin, Y. Li, R. Weiss, R. D. Kamm, C. S. Chen, and H. H. Asada. Formation and optogenetic control of engineered 3D skeletal muscle bioactuators. Lab Chip, 12(23):4976-4985, Dec. 2012.

47. J. Schindelin, I. Arganda-Carreras, E. Frise, V. Kaynig, M. Longair, T. Pietzsch, S. Preibisch, C. Rueden, S. Saalfeld, B. Schmid, J.-Y. Tinevez, D. J. White, V. Hartenstein, K. Eliceiri, P. Tomancak, and A. Cardona. Fiji: an open-source platform for biological-image analysis. Nat. Methods, 9(7):676-682, June 2012.

48. A. Shima, Y. Morimoto, H. L. Sweeney, and S. Takeuchi. Three-dimensional contractile muscle tissue consisting of human skeletal myocyte cell line. Exp. Cell Res., 370(1):168-173, 2018.

49. A. S. T. Smith, C. J. Long, K. Pirozzi, S. Najjar, C. McAleer, H. H. Vandenburgh, and J. J. Hickman. A multiplexed chip-based assay system for investigating the functional development of human skeletal myotubes in vitro. $J$. Biotechnol., 185:15-18, Sept. 2014.

50. E. J. Stevenson, A. Koncarevic, P. G. Giresi, R. W. Jackman, and S. C. Kandarian. Transcriptional profile of a myotube starvation model of atrophy. $J$. Appl. Physiol., 98(4):1396-1406, Apr. 2005.

51. H. Takahashi, T. Shimizu, and T. Okano. Engineered Human Contractile Myofiber Sheets as a Platform for Studies of Skeletal Muscle Physiology. Sci Rep, 8(1):13932, 2018. 
52. M. W. Toepke and D. J. Beebe. PDMS absorption of small molecules and consequences in microfluidic applications. Lab Chip, 6(12):1484-1486, Dec. 2006.

53. N. Träber, K. Uhlmann, S. Girardo, G. Kesavan, K. Wagner, J. Friedrichs, R. Goswami, K. Bai, M. Brand, C. Werner, D. Balzani, and J. Guck. Polyacrylamide Bead Sensors for in vivo Quantification of Cell-Scale Stress in Zebrafish Development. Sci Rep, 9(1):17031, Nov. 2019.

54. H. Vandenburgh, J. Shansky, F. Benesch-Lee, V. Barbata, J. Reid, L. Thorrez, R. Valentini, and G. Crawford. Drug-screening platform based on the contractility of tissue-engineered muscle. Muscle Nerve, 37(4):438-447, Apr. 2008.

55. D. Vorselen, Y. Wang, M. M. de Jesus, P. K. Shah, M. J. Footer, M. Huse, W. Cai, and J. A. Theriot. Microparticle traction force microscopy reveals subcellular force exertion patterns in immune cell-target interactions. Nat Commun, 11(1):20, Jan. 2020.

56. C. L. Walker, K. A. Lukyanov, I. V. Yampolsky, A. S. Mishin, A. S. Bommarius, A. M. Duraj-Thatte, B. Azizi, L. M. Tolbert, and K. M. Solntsev. Fluorescence imaging using synthetic GFP chromophores. Curr Opin Chem Biol, 27:64-74, Aug. 2015.

57. B. Wallmeyer, S. Trinschek, S. Yigit, U. Thiele, and T. Betz. Collective Cell Migration in Embryogenesis Follows the Laws of Wetting. Biophys. J., 114(1):213-222, 2018.

58. B. Wallmeyer, Bernhard. Bead Segmentation. https://github.com/tobetz/ElasticBeadAnalysis, Jan. 2020. 